\title{
Spin Dependent Fano Resonances in a Rashba System with Coulomb Interactions
}

\author{
P. STEFAŃSKI* \\ Institute of Molecular Physics of the Polish Academy of Sciences \\ M. Smoluchowskiego 17, 60-179 Poznań, Poland
}

\begin{abstract}
The effects of interplay of interference of quantum mechanical electron waves and their mutual Coulomb interactions are investigated in the device composed of interacting quantum dot attached to polarized leads via quantum point contacts with the Rashba interaction. The Zeeman-split dot sub-levels form two interfering channels and as a result spin dependent Fano resonances arise in the conductance through the system. The Coulomb repulsion between the channels modifies the width and shape of the Fano resonances as compared to the non-interacting case. We formulate the Fano expression dependent on the dot's occupancy regulated by the Coulomb interactions.
\end{abstract}

PACS: 73.23.-b, 72.25.Dc, 73.40.Sx

\section{Introduction}

Depending on the kind of experiment electrons exhibit particle or quantum-wave nature. Typical quantum interference experiments, for instance, are performed in the Aharonov-Bohm geometry, where the phase of electron waves can be controlled by the external magnetic field [1]. But electrons also possess spin and are charged particles, which is manifested in experiments as the Coulomb blockade and the Kondo effect observed in the transport through quantum dots [2].

\section{Model}

An interesting question arises whether electron correlations affect interference of quantum mechanical electron waves. To answer this question we took into account a small, interacting quantum dot (QD) with one spatial level. The dot is coupled to spin-polarized metallic leads via quantum point contacts (QPCs) with the Rashba interaction [3] arising from the strong asymmetry of the nanostructure confining potential in $y$-direction

$$
H_{\mathrm{R}}=\frac{\alpha}{2 \hbar} \hat{y} \cdot(\hat{\sigma} \times \hat{p})=\frac{\alpha}{2 \hbar}\left(\sigma_{z} p_{x}-\sigma_{x} p_{z}\right) .
$$

We have assumed that the Rashba coefficient $\alpha$ does not depend on $x$-direction of electron tunneling. Inter-subband mixing, described by the second term of Eq. (1), introduces quantum interference between electron waves of opposite spin densities [4]. Inside the dot they mutually interact via the Coulomb repulsion. Thus, quantum interference takes place between spin up $\epsilon_{d \uparrow}$ and

\footnotetext{
* e-mail: piotrs@ifmpan.poznan.pl
}

spin down $\epsilon_{d \downarrow}$ channels in presence of their Coulomb interaction, producing the Fano resonances [5]. We assume that the inter-subband mixing in QPCs dominates over the spin precession first term. It is realized, when the ratio of $\mathrm{QPC}$ length to width $L / W \approx 1$ and the $\mathrm{QPC}$ confinement energy (in our case in $z$-direction) is comparable to the spin splitting energy introduced by the Rashba effect. The Rashba effect inside QPCs is then written in the form of tight-binding Hamiltonian [6] as two hopping matrix elements between the dot and the leads: $t^{\alpha}$ - "direct", spin-conserved tunneling and $t_{\mathrm{SO}}^{\alpha}$ "indirect" spin-flip tunneling, mediated by inter-subband mixing:

$$
\begin{aligned}
& H_{\mathrm{tun}}=\sum_{k, \sigma, \alpha=\mathrm{L}, \mathrm{R}}\left[t^{\alpha} c_{k \alpha, \sigma}^{\dagger} d_{\sigma}+t_{\mathrm{SO}}^{\alpha} c_{k \alpha, \bar{\sigma}}^{\dagger}\left(i \sigma_{x}\right)_{\sigma \bar{\sigma}} d_{\sigma}\right. \\
& \quad+\text { H.c. }] .
\end{aligned}
$$

The complex $\mathrm{i}$ factor in the Rashba term ensures its time reversal invariance, which is a feature of the Rashba Hamiltonian, Eq. (1). The parts of the Hamiltonian describing the leads and $\mathrm{QD}$ are: $H_{\text {Leads }}+H_{\mathrm{QD}}=$ $\sum_{k, \sigma, \alpha=\mathrm{L}, \mathrm{R}} \epsilon_{k \alpha, \sigma} c_{k \alpha, \sigma}^{\dagger} c_{k \alpha, \sigma}+\sum_{\sigma} \epsilon_{d} d_{\sigma}^{\dagger} d_{\sigma}+U n_{\sigma} n_{\bar{\sigma}}$.

Considered system is exposed to an external Zeeman field in the $z$-direction: $\Delta=\epsilon_{d \uparrow}-\epsilon_{d \downarrow}, \Delta=|g| \mu_{\mathrm{B}} H$. We neglect a possible influence of magnetic field on the electron phase because $\epsilon_{d \uparrow}$ and $\epsilon_{d \downarrow}$ are spatially overlapping.

The dot's sublevels are uniformly shifted by gate voltage acting on the dot capacitatively: $\epsilon_{d \sigma} \equiv \epsilon_{d \sigma}-V_{\mathrm{g}}$, and for $\Delta=0$ the dot's level initial position is assumed to coincide with the Fermi level placed at zero energy $\epsilon_{d}=\epsilon_{\mathrm{F}}=0$.

Let us define the polarization of the lead $\alpha$ as a relative difference between spectral densities of the lead subbands of spin up and down: $P_{\alpha}=\left(\rho_{\alpha \uparrow}-\rho_{\alpha \downarrow}\right) /\left(\rho_{\alpha \uparrow}+\rho_{\alpha \downarrow}\right)$. 
It can be expressed by the spin-dependent QD level width acquired by the "direct" tunneling: $P_{\alpha}=\left(\Gamma_{\alpha \uparrow}-\right.$ $\left.\Gamma_{\alpha \downarrow}\right) /\left(\Gamma_{\alpha \uparrow}+\Gamma_{\alpha \downarrow}\right)$.

Presence of the Rashba interaction inside QPCs introduces two additional transmission spin-flip channels besides diagonal $\epsilon_{\uparrow}$ and $\epsilon_{\downarrow}$ channels. We then write the retarded Green function of the QD and the current in a matrix form in spin indices

$$
\hat{G}^{\mathrm{r}}=\left[\begin{array}{cc}
G_{\uparrow \uparrow}^{\mathrm{H}}(\omega)^{-1} & -\frac{1}{2} \sum_{\alpha} \Gamma_{\uparrow \downarrow}^{\alpha} \\
\frac{1}{2} \sum_{\alpha} \Gamma_{\uparrow \downarrow}^{\alpha} & G_{\downarrow \downarrow}^{\mathrm{H}}(\omega)^{-1}
\end{array}\right]^{-1} .
$$

The $\hat{\Gamma}^{\alpha}$ matrix describing coupling of the dot to the $\alpha$ lead reads

$$
\hat{\Gamma}^{\alpha}=\left[\begin{array}{cc}
\Gamma_{\uparrow \uparrow}^{\alpha} & \mathrm{i} \Gamma_{\uparrow \downarrow}^{\alpha} \\
-\mathrm{i} \Gamma_{\uparrow \downarrow}^{\alpha} & \Gamma_{\downarrow \downarrow}^{\alpha}
\end{array}\right],
$$

where $\Gamma_{\sigma \sigma}^{\alpha}=\Gamma_{\alpha \sigma}+\Gamma_{\alpha \bar{\sigma}}^{\mathrm{SO}}$ and $\Gamma_{\sigma \bar{\sigma}}^{\alpha}=\sqrt{\Gamma_{\alpha \sigma} \Gamma_{\alpha \sigma}^{\mathrm{SO}}}-$ $\sqrt{\Gamma_{\alpha \bar{\sigma}} \Gamma_{\alpha \bar{\sigma}}^{\mathrm{SO}}}$. The level widths $\Gamma_{\alpha \sigma}=2 \pi\left|t^{\alpha}\right|^{2} \rho_{\alpha \sigma}$ and $\Gamma_{\alpha \sigma}^{\mathrm{SO}}=2 \pi\left|t_{\mathrm{SO}}^{\alpha}\right|^{2} \rho_{\alpha \sigma}$ have usual form of a single channel case.

The diagonal elements in Eq. (3) are inverse of the dot Green functions calculated in the Hubbard approximation [7, 8], which is most suitable for the description of QD level in the Coulomb blockade regime. For spin $\sigma$ it has the form

$$
\begin{aligned}
& G_{\sigma \sigma}^{\mathrm{H}}(\omega)=\left(\frac{\omega-\epsilon_{d \sigma}}{1+\frac{\left\langle n_{\bar{\sigma}}\right\rangle}{\omega-\epsilon_{d \sigma}-U}}+\frac{\mathrm{i}}{2} \sum_{\alpha} \Gamma_{\sigma \sigma}^{\alpha}\right)^{-1} \\
& \simeq \frac{1-\left\langle n_{\bar{\sigma}}\right\rangle}{\omega-\epsilon_{d \sigma}+\frac{\mathrm{i}}{2} \Gamma_{\sigma \sigma}^{\sigma}}+\frac{\left\langle n_{\bar{\sigma}}\right\rangle}{\omega-\epsilon_{d \sigma}-U+\frac{\mathrm{i}}{2} \Gamma_{\sigma \sigma}^{\sigma}} .
\end{aligned}
$$

Equation (5) has been written as the sum of two Hubbard resonances, $\epsilon_{d \sigma}^{\mathrm{I}} \equiv \epsilon_{d \sigma}$ and $\epsilon_{d \sigma}^{\mathrm{II}} \equiv \epsilon_{d \sigma}+U$, whose spectral weights are controlled by the occupancy with the opposite spin $\bar{\sigma}$. This feature, caused by the Coulomb interactions significantly modifies the Fano resonance as compared to single particle case [5].

The occupancies $\left\langle n_{\uparrow}\right\rangle$ and $\left\langle n_{\downarrow}\right\rangle$ have been calculated selfconsistently from the set of coupled equations, similarly as in [8], from the diagonal elements of the "lesser" Green function matrix: $\hat{G}^{<}=\hat{G}^{\mathrm{r}} \hat{\Sigma}^{<} \hat{G}^{\mathrm{a}}$ with the "lesser" self-energy describing the coupling to the leads in both spin sectors.

The current through the device is calculated from the Meir-Wingreen expression [9], but written in the spin space for symmetric coupling $\hat{\Gamma}^{\mathrm{L}}=\hat{\Gamma}^{\mathrm{R}} \equiv \hat{\Gamma}$ :

$$
\mathcal{G}=\frac{e^{2}}{2 h} \int \mathrm{d} \epsilon\left(-\frac{\partial f(\epsilon)}{\partial \epsilon}\right) \mathrm{i} \operatorname{Tr}\left\{\hat{\Gamma}\left[\hat{G}^{\mathrm{r}}(\epsilon)-\hat{G}^{\mathrm{a}}(\epsilon)\right]\right\} .
$$

The summation over spins is already included in the trace.

In numerical calculations we assumed broad, featureless density of states in the leads $\rho_{\alpha \sigma}=(1 / D) \Theta(|\epsilon|-D)$. We have set Coulomb interaction in the $\operatorname{dot} U=0.1 D$, $t^{\alpha}=0.8 U$ and $t_{\mathrm{SO}}^{\alpha} / t^{\alpha}=0.5$ for $\alpha=\mathrm{L}, \mathrm{R}$.

\section{Results and discussion}

In Fig. 1 we show interaction-only $\left(t_{\mathrm{SO}}^{\alpha}=0\right)$ conductance for various Zeeman splitting, calculated with the use of diagonal Meir-Wingreen expression [8]. We set $P_{\mathrm{L}}=P_{\mathrm{R}}=0.6$, which gives the same diagonal broadenings $\Gamma_{\uparrow \uparrow}^{\alpha}=0.4 U$ and $\Gamma_{\downarrow \downarrow}^{\alpha}=0.1 U$ as in the Rashba case for $t_{\mathrm{SO}}^{\alpha}=0.5 t^{\alpha}$ and $P_{\mathrm{L}}=P_{\mathrm{R}}=1$. For $\Delta=0$, part (a), zero bias conductance displays usual two Hubbard peaks for single and double electron occupancy, separated by $U$. Conductance peaks reach unitary limit at $V_{\mathrm{g}}=0$ and $V_{\mathrm{g}}=U$ when the Hubbard levels $\epsilon_{\sigma}^{\mathrm{I}}=\epsilon_{\mathrm{F}}$ and $\epsilon_{\sigma}^{\mathrm{II}}=\epsilon_{\mathrm{F}}$, respectively. The conductance curve is symmetric with respect to the particle-hole symmetry line at $V_{\mathrm{g}}=U / 2$ $\left(\epsilon_{\uparrow}=\epsilon_{\downarrow}=-U / 2\right)$. Let us note also that $\left\langle n_{\uparrow}\right\rangle$ and $\left\langle n_{\downarrow}\right\rangle$ vs. gate voltage shown in part (a) do not coincide because there is finite polarization of the leads [8].

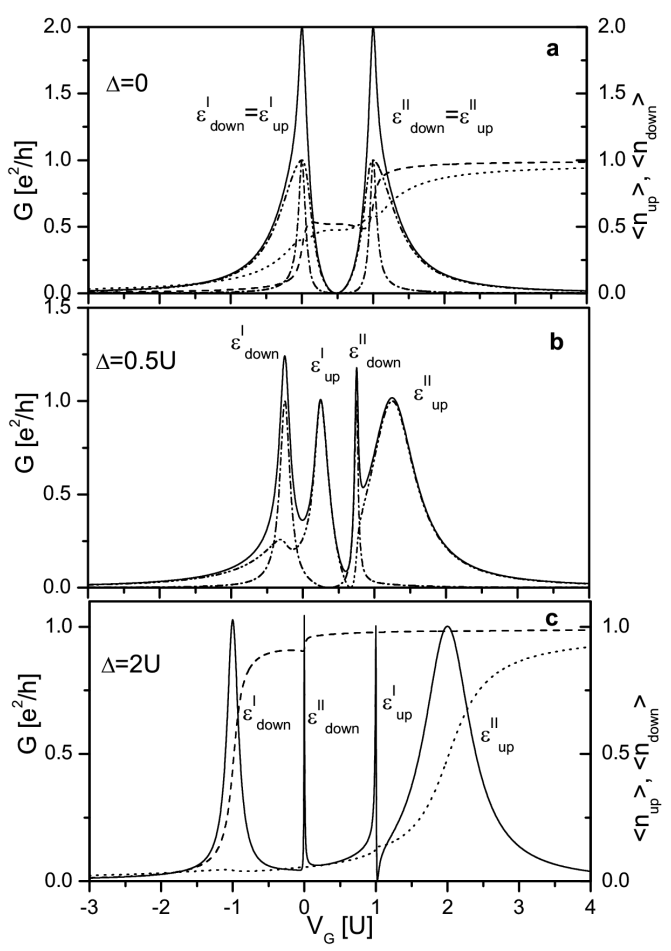

Fig. 1. Interaction-only zero bias conductance, its spin components and $\left\langle n_{\downarrow}\right\rangle$ and $\left\langle n_{\uparrow}\right\rangle$ occupancies vs. gate voltage, for leads polarization $P_{\mathrm{L}}=P_{\mathrm{R}}=0.6$ and various Zeeman splitting $\Delta$. (a) For $\Delta=0$; solid curve - total conductance, dash-dot-dotted curve $-\mathcal{G}_{\uparrow}$, dashed-dotted curve $-\mathcal{G}_{\downarrow},\left\langle n_{\uparrow}\right\rangle$ - dotted curve, $\left\langle n_{\downarrow}\right\rangle-$ dashed curve. (b) $\mathcal{G}, \mathcal{G}_{\uparrow}$ and $\mathcal{G}_{\downarrow}$ for $\Delta=0.5 U$; symbols the same as in (a). (c) Curves for $\Delta=2 U$, symbols the same as in (a). The conductance peaks are associated with corresponding $\epsilon_{\sigma}^{\gamma}$ Hubbard resonances, $\sigma=\uparrow$ or $\downarrow$ and $\gamma=$ I or II.

For finite Zeeman splitting, as shown in parts (b) and (c) of Fig. 1, the symmetry of conductance no longer persists. The first and second Hubbard resonance in each spin sector have very different spectral weights due to the different dependence of occupancies vs. gate voltage, 
see $\left\langle n_{\uparrow}\right\rangle$ and $\left\langle n_{\downarrow}\right\rangle$ for $\Delta=2 U$ in part (c). These features, caused by electron interactions, have profound effect on the formation of the Fano resonances. Let us now switch on the Rashba interaction in the QPCs. Now inter-subband mixing opens new, spin-flip transmission channels. Quantum interference between $\epsilon_{\downarrow}$ and $\epsilon_{\uparrow}$ takes place and they take the role of interfering channels characteristic for the Fano resonance: the $\epsilon_{\downarrow}$ of resonant level and the $\epsilon_{\uparrow}$ of broad, background continuum. In presence of electron interactions they evolve into the Hubbard resonances and now quantum interference takes place between them $\epsilon_{d \downarrow}^{\gamma} \leftrightarrow \epsilon_{d \uparrow}^{\gamma}, \gamma=$ I or II. Indeed, the expression for conductance, Eq. (6), can be approximated by a product of the Fano expression for resonance at $\epsilon_{d \downarrow}^{\gamma}=\epsilon_{\mathrm{F}}$ and conductance through the "background" Hubbard resonance $\epsilon_{d \uparrow}^{\gamma}$. At $T=0$ and for $P_{\mathrm{L}}=P_{\mathrm{R}}=1$ it can be written

$$
\begin{aligned}
& \mathcal{G}^{\text {int }}\left(\epsilon_{\mathrm{F}}\right) \simeq \frac{e^{2}}{h} \sum_{\gamma=\mathrm{I}, \mathrm{II}} \mathcal{G}_{\uparrow}^{\gamma}\left(\epsilon_{\mathrm{F}}\right) \frac{\left(\tilde{\epsilon}_{d \downarrow}^{\gamma}+q^{\gamma}\right)^{2}}{\left(\tilde{\epsilon}_{d \downarrow}^{\gamma}\right)^{2}+1}, \\
& \mathcal{G}_{\uparrow}^{\gamma}\left(\epsilon_{\mathrm{F}}\right)=\frac{\left(\tilde{\Gamma}_{\uparrow}^{2}\right.}{\left(\epsilon_{\mathrm{F}}-\epsilon_{d \uparrow}^{\gamma}\right)^{2}+\left(\tilde{\Gamma}_{\uparrow}^{\gamma}\right)^{2}}, \\
& \tilde{\epsilon}_{d \downarrow}^{\gamma}=\frac{\epsilon_{\mathrm{F}}-\epsilon_{d \downarrow}^{\gamma}}{\tilde{\Gamma}_{\uparrow}^{\gamma, \mathrm{SO}},} \quad q^{\gamma}=\frac{\epsilon_{\mathrm{F}}-\epsilon_{d \uparrow}^{\gamma}}{\tilde{\Gamma}_{\uparrow}^{\gamma}}, \\
& \tilde{\Gamma}_{\uparrow}^{\gamma}=\alpha_{\uparrow}^{\gamma} \Gamma_{\uparrow}, \quad \tilde{\Gamma}_{\uparrow}^{\gamma, \mathrm{SO}}=\alpha_{\downarrow}^{\gamma} \Gamma_{\uparrow}^{\mathrm{SO}}, \\
& \alpha_{\sigma}^{\gamma}= \begin{cases}1-\left\langle n_{\bar{\sigma}}\right\rangle, & \gamma=\mathrm{I}, \\
\left\langle n_{\bar{\sigma}}\right\rangle, & \gamma=\mathrm{II} .\end{cases}
\end{aligned}
$$

We defined reduced resonance level $\tilde{\epsilon}_{d \downarrow}$ and the Fano asymmetry parameter $q$. For $P_{\mathrm{L}}=P_{\mathrm{R}}=1$ and symmetric coupling diagonal broadenings are $\Gamma_{\uparrow \uparrow}^{\alpha}=\Gamma_{\alpha \uparrow} \equiv \Gamma_{\uparrow}$ and $\Gamma_{\downarrow \downarrow}^{\alpha}=\Gamma_{\alpha \uparrow}^{\mathrm{SO}} \equiv \Gamma_{\uparrow}^{\mathrm{SO}}$. Presence of electron interactions manifests in Eq. (7) as a level width renormalization $\tilde{\Gamma}_{\uparrow}^{\gamma}$ and $\tilde{\Gamma}_{\uparrow}^{\gamma, \mathrm{SO}}$ by the dot occupancies. Through this renormalization they also modify the Fano $q$ parameter. For non-interacting case $\alpha_{\sigma}^{\gamma} \equiv 1$ and one considers one pair of interfering single particle channels replacing appropriate Hubbard resonances.

In Fig. 2 we show numerical results for the conductance calculation at $T=0, P_{\mathrm{L}}=P_{\mathrm{R}}=1$ and $t_{\mathrm{SO}}^{\alpha}=0.5 t_{\alpha}$ for various Zeeman splitting. Conductance curves are considerably modified by quantum interference as compared to results in Fig. 1. For $\Delta=0.5 U$, in part (a), the conductance peaks associated with the Hubbard levels of spin down sector transform into the Fano resonances and zeros of the conductance appear. Conductance calculated for the same Zeeman splitting but non-interacting dot is shown by dotted curve. The Fano resonances labeled by $\epsilon_{\downarrow}^{\mathrm{I}}$ nearly coincide in interacting and non-interacting cases, while the spectral weight of the background channel, labeled by $\epsilon_{\uparrow}^{\mathrm{I}}$ has been changed by electron interactions.

This is a remarkable feature of the Fano resonance in presence of electron interactions, that the background conductance channel can become even sharper than the resonant channel. It is better seen for $\Delta=2 U$, in

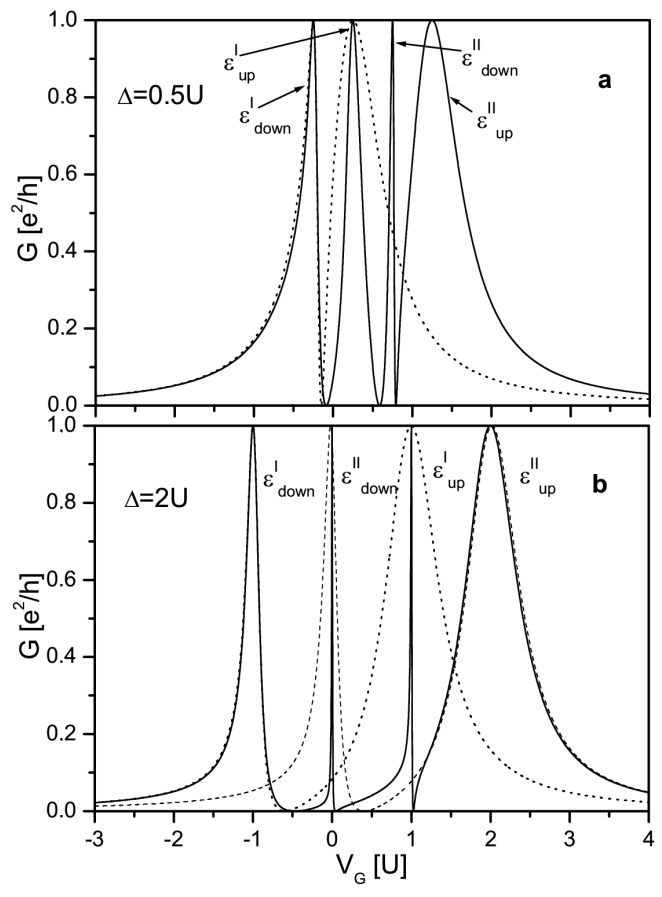

Fig. 2. Interference-and-interaction zero bias conductances vs. gate voltage calculated for $t_{\alpha}^{\mathrm{SO}}=0.5 t_{\alpha}$, $P_{\mathrm{L}}=P_{\mathrm{R}}=1$ and various Zeeman splitting $\Delta$. (a) for $\Delta=0.5 U$; solid curve - conductance, dotted curve - conductance for non-interacting case. (b) Conductances for $\Delta=2 U$; additionally dashed curve - conductance for non-interacting case when resonant level and background channel placed at $\epsilon_{\downarrow}^{\mathrm{II}}$ and $\epsilon_{\uparrow}^{\mathrm{II}}$ Hubbard resonances, respectively.

part (b), where the background channel labeled by $\epsilon_{\uparrow}^{\mathrm{I}}$ became much sharper than the Fano resonance labeled by $\epsilon_{\downarrow}^{\mathrm{I}}$. Let us note that the shape of $\epsilon_{\uparrow}^{\mathrm{I}}$ peak resembles the Fano resonance, but it is not the case. The asymmetry of this peak is introduced by electron interactions alone in presence of finite Zeeman splitting, as shown in Fig. 1c. The Fano resonance labeled by $\epsilon_{\downarrow}^{I}$ in part (b) has asymmetry parameter $q=-\tilde{\epsilon}_{\uparrow} / \Gamma_{\uparrow}=-5$ for non-interacting dot, while in presence of interactions $q^{\mathrm{I}}=-\tilde{\epsilon}_{\uparrow}^{\mathrm{I}} / \tilde{\Gamma}_{\uparrow}=-8.97$.

The main difference in shape, which is the maximum of the Fano resonance, is blurred by the convolution with the conductance $\mathcal{G}_{\uparrow}$ through background channel, Eq. (7). Another Fano resonance appears at $V_{\mathrm{g}}=0$ when $\epsilon_{\downarrow}^{\mathrm{II}}=\epsilon_{\mathrm{F}}$, due to quantum interference with the background channel located at $\epsilon_{\uparrow}^{\mathrm{II}}$. Dashed curve is conductance calculated for non-interacting QD for the same level arrangement. For interacting case the Fano resonance is much narrower due to the fact that its width is renormalized by interactions $\tilde{\Gamma}_{\uparrow}^{\mathrm{SO}}=0.006 \mathrm{U}$, whereas without interactions $\Gamma_{\uparrow}^{\mathrm{SO}}=0.1 U$. Asymmetry parameters are respectively $q^{\mathrm{II}}=-5.46$ and $q=-5$. Non-interacting Fano resonances placed at $\epsilon_{\downarrow}^{\gamma}, \gamma=$ I or II have the same $q$ parameters because the distance in energy scale between 
them and background channels placed at $\epsilon_{\uparrow}^{\gamma}$ is the same, equal to $\Delta$ and the width of the background channel is constant in this case.

\section{Acknowledgments}

This work was supported by Ministry of Science and Higher Education (Poland) from sources for science in the years 2009-2012 within a research project.

\section{References}

[1] R. Schuster, E. Buks, M. Heiblum, D. Mahalu, V. Umansky, H. Shtrikman, Nature (London) 385, 417 (1987).

[2] D. Goldhaber-Gordon, H. Shtrikman, D. Mahalu, D. Abusch-Magder, U. Meirav, M.A. Kastner, Nature (London) 391, 156 (1998).
[3] Y.A. Bychkov, E.I. Rashba, J. Phys. C 17, 6039 (1984).

[4] D. Sánchez, L. Sierra, Phys. Rev. B 74, 153313 (2006).

[5] U. Fano, Phys. Rev. 124, 1866 (1961).

[6] Q. Sun, J. Wang, H. Guo, Phys. Rev. B 71, 165310 (2005).

[7] A.C. Hewson, Phys. Rev. 144, 420 (1966).

[8] P. Stefański, Phys. Rev. B 77, 125331 (2008).

[9] Y. Meir, N.S. Wingreen, Phys. Rev. Lett. 68, 2512 (1992). 